\title{
Founder effect in spinal and bulbar muscular atrophy (SBMA) in Scandinavia
}

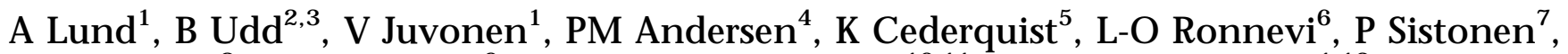 \\ SA Sörensen ${ }^{8}, L_{\text {Tranebjærg }}{ }^{9}, C$ Wallgren-Pettersson ${ }^{10,11}$ and M-L Savontaus ${ }^{1,12}$
}

\begin{abstract}
${ }^{1}$ Department of Medical Genetics, University of Turku; ${ }^{2}$ Neurological Department, Vasa Central Hospital, Vasa; ${ }^{3}$ Department of N eurology, University of Tampere, Finland; Departments of ${ }^{4} \mathrm{~N}$ eurol ogy and ${ }^{5} \mathrm{M}$ edical Genetics, University of Umeå; ${ }^{6}$ D epartment of Neurology, Karolinska Sjukhuset, Stockholm, Sweden; ${ }^{7}$ Finnish Red Cross, Blood Transfusion Service, Helsinki, Finland; ${ }^{8}$ D epartment of Medical Genetics, University of Copenhagen, Denmark; ${ }^{9}$ Department of Medical Genetics, University Hospital of Tromsö, Norway; ${ }^{10}$ The Folkhälsan Department of Medical Genetics, Helsinki; ${ }^{11}$ Department of Medical Genetics, University of Helsinki; ${ }^{12}$ Department of Biology, University of Turku, Finland
\end{abstract}

We haplotyped 13 Finnish, 10 Swedish, 12 Danish and 2 Norwegian SBMA (spinal and bulbar muscular atrophy, Kennedy disease) families with a total of 45 patients and 7 carriers for 17 microsatellite markers spanning a $25.2 \mathrm{cM}$ region around the androgen receptor gene on chromosome Xq11-q12 in search of a genetic founder effect. In addition, the haplotypes of 50 Finnish, 20 Danish and 22 Swedish control males were examined. All the Scandinavian SBMA families shared the same 18 repeat allele for the intragenic GGC repeat, which was present in only $24 \%$ of the controls. Linkage disequilibrium was also seen for the closest microsatellite markers. In addition, extended haplotypes of the Finnish, Swedish and Danish SBMA families revealed country-specific common founder haplotypes, which over time became gradually shortened by recombinations. No common haplotype was found among the controls. The data suggest that the SBMA mutation was introduced into western Finland 20 generations ago. Haplotype analysis implies a common ancestor for the majority of Scandinavian SBMA patients. European Journal of Human Genetics (2000) 8, 631-636.

Keywords: SBMA; Kennedy disease; trinucleotide repeat; CAG repeat disorder; founder effect

\section{Introduction}

Spinal and bulbar muscular atrophy (SBMA), or Kennedy disease (MIM 313200), is an X-linked recessive late-onset motor neurone disorder. Patients show slowly progressive spinal and bulbar muscular atrophy with fasciculations and generalised muscle weakness, frequently sensory symptoms and signs of partial androgen insensitivity, such as gynecomastia and impotence. ${ }^{1,2}$ SBMA is caused by a CAG repeat expansion in the first exon of the androgen receptor (AR) gene. ${ }^{3}$ The gene normally has $10-36$ CAG repeats, ${ }^{4}$ but in SBMA patients the number of repeats is increased to 38-72. 5,6 Several other neurodegenerative disorders caused by trinucleotide repeat expansions have also been reported. ${ }^{7-9}$ It has

Correspondence: Annastiina Lund, Department of Medical Genetics, University of Turku, Kiinamyllynkatu 10, FIN-20520 Turku, Finland. Tel: + 3582333 7456; Fax: + 3582333 7300; E-mail: anniemi@utu.fi Received 26 January 2000; revised 26 April 2000; accepted 27 April 2000 been shown that proteins with expanded polyglutamine stretches encoded by the CAG repeats form fibrillary aggregates in neurones, leading to apoptosis. ${ }^{10}$ Such aggregates have also been reported in SBMA., ${ }^{4,6,11}$

The human AR gene is located in Xq11-q12. The gene contains 8 exons. ${ }^{3}$ In the $3^{\prime}$ end of the first exon of the AR gene, $1.2 \mathrm{~kb}$ downstream from the CAG repeat there is a polymorphic GGC repeat sequence. ${ }^{12}$ In Japanese SBMA patients linkage disequilibrium between the CAG mutation and the intragenic GGC repeat has been found, indicating a founder effect. ${ }^{13}$ The high prevalence of SBMA among Swedish-speaking people in the district of Vaasa in Western Finland ${ }^{2}$ and in Northern Sweden (6 in 930000 ) motivated us to search for linkage disequilibrium between the mutation and 17 microsatellite markers around the AR gene, and also to estimate the time when the SBMA mutation was introduced into Finland. Our initial studies showed that all 
13Finnish families shared identical alleles for at least five adjacent markers. This finding prompted us to extend the study to patients from the linguistically related neigh bouring Scandinavian countries in the search for a genetic founder effect.

\section{Material and methods}

Thirteen Finnish families (FI-FXIII) with a total of 17SBMA patients and four carriers, 10Swedish families (SI-SX) with 13 patients, 12Danish patients from different families (DI-DXII) and two Norwegian families (NI and NII) with three patients and three carriers were haplotyped for 17 microsatellite markers spanning a $25.2 \mathrm{cM}$ region around the androgen receptor gene (Figure 1 ). The locations and distances of the markers were obtained from The Genome Database (GDB) (http://gdbwww.gdb.org/). The haplotypes of 50 Finnish, 20Danish and 22 Swedish control males were also examined, as well as the CAG repeat numbers in 25 Finnish control women.

After obtaining informed consent, blood samples were taken of patients with SBMA. DNA was extracted from EDTA

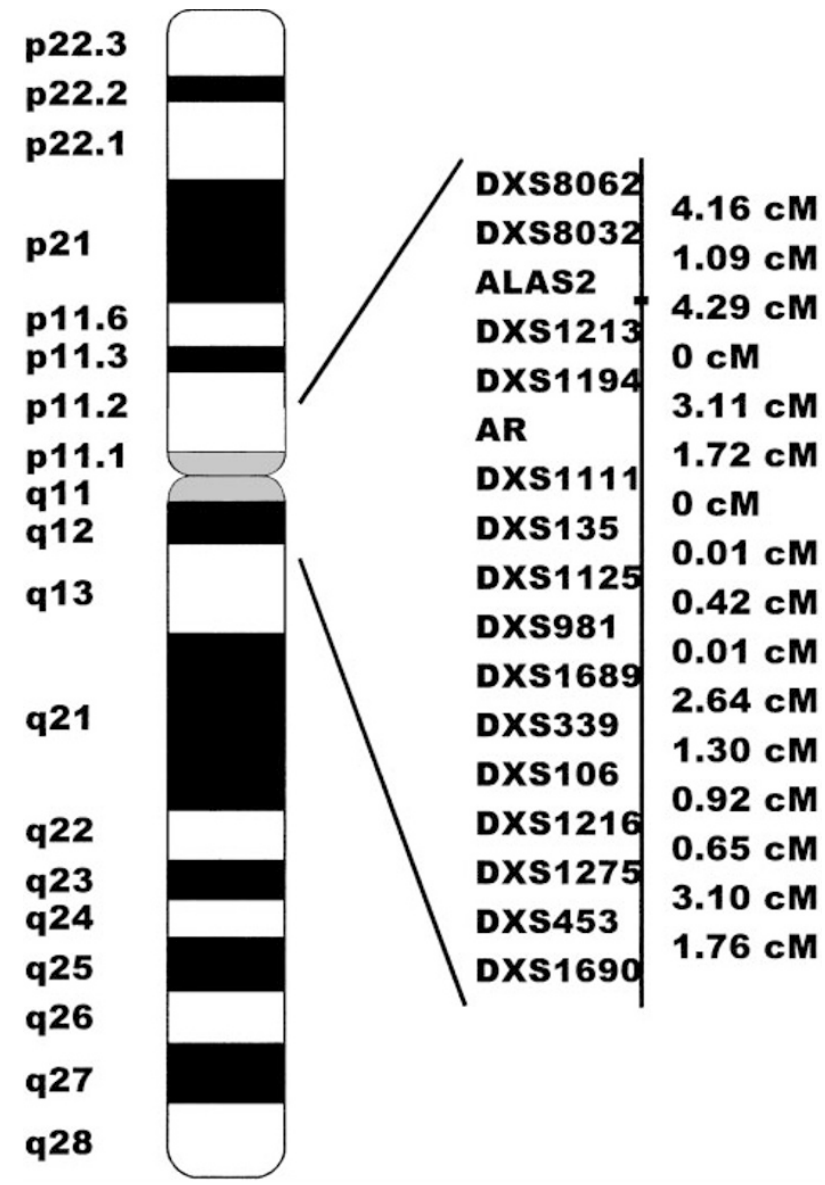

Figure 1 Genetic distances of the microsatellite markers and their locations on the X chromosome according to GDB. blood. The primers for the microsatellites were from the GDB Polymorphism Detail, except for the $\mathrm{CAG}^{3}$ and the $\mathrm{GGC}^{13}$ repeats in the AR gene. The microsatellites were amplified by a standard PCR method with $50 \mathrm{ng}$ of template DNA, $10 \mathrm{pmol}$ of each primer, $200 \mu \mathrm{M}$ of dNTP, $1 \times$ PCR buffer (Finnzymes),

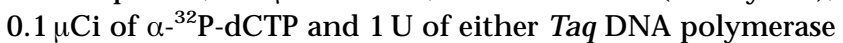
(Promega) (CAG, GGC, ALAS2, DXS8032, DXS8062, DXS339 and DXS1213) or Dynazyme DNA polymerase (Finnzymes). In addition, the reactions for CAG and DXS8032 contained $10 \%$ glycerol, and CAG contained $2 \%$ formamide. For the GGC repeat, 7-deaza-dGTP was included in the dNTP mix, and $10 \%$ DMSO was used. The reaction volumes were $25 \mu \mathrm{l}$, except for GGC, in which volume was $15 \mu \mathrm{l}$. The initial denaturation was at $95^{\circ} \mathrm{C}$ for $5 \mathrm{~min}$, denaturation at $95^{\circ} \mathrm{C}$, annealing at $50-64^{\circ} \mathrm{C}$, elongation at $72^{\circ} \mathrm{C}$ and a final elongation at $72^{\circ} \mathrm{C}$ for $4 \mathrm{~min}$. Denaturation, annealing and elongation were $45 \mathrm{~s}$ each, except for CAG 1 min each and for GGC 1, 2 and 2 min, respectively. Thirty-five cycles were used for DXS1690, while the others went through 30 cycles. The sizes of the PCR products were analysed by comparison with an M 13 DNA sequencing ladder in 4-6\% denaturing PAGE gels. The CAG and GGC repeat numbers were verified by comparison with sequenced references.

The allele frequencies of the SBMA patients and the controls were compared using the Mann-Whitney test, and the CAG repeat ranges of the Finnish, Swedish and Danish patients were compared by variance analysis.

A tentative age estimate in the Finnish sample based on the number of SBMA chromosomes, linkage disequilibrium observed, recombination fractions obtained from GDB and assumptions on ascertainment was made using the DMLE program (version 0.25). ${ }^{14}$ We assumed complete isolation of the Finnish Swedish-speaking coastal population and a population growth equal to that generally estimated for Finland from mediaeval times (population base of about 100000 ) from around year 1500 until the present (population base of about 5 million). As the algorithm carrying out the Monte Carlo replicates in DMLE only applies to marker loci with two allelic variants, only two loci were considered in the analysis, ALAS2 and DXS1125, the closest recombinant flanking loci to AR, both showing biallelic segregation in the Finnish SBMA families. For each log likelihood, a million Monte Carlo replicates were computed using a generation interval of 2. X-chromosomal inheritance was allowed for by simply halving the recombination fractions.

\section{Results}

All the Finnish SBMA families shared an identical haplotype 2-3-18-2-4 for the loci DXS1213-DXS1194-AR GGCDXS1111-DXS135, spanning $4.8 \mathrm{cM}$ calculated from GDB, and all except FV had the same DXS1213-DXS981 haplotype 2-3-18-2-4-6-7 +, spanning 5.3 cM (GDB) (Table1). Nine out of ten Swedish SBMA families had the same DXS1213DXS135 haplotype as most of the Finnish families. Ten out of 
Table 1 The haplotypes of the Finnish, Swedish, Danish and Norwegian SBMA families and the base-pair sizes of the alleles. The common haplotypes shared by SBMA families in each country are shaded. The number behind the family symbol stands for the individual in the family. When there is no number after the symbol, all the examined family members shared the same haplotype. Different CAG numbers in families FIII, FV and NII are shown by multiple CAG numbers. *DXS981 is a tetranucleotide combined with deletions. + and - stand for alleles that are longer or shorter by one nucleotide, eg 7+=188 bp and 6-=189 bp

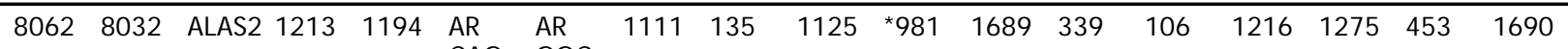

\begin{tabular}{lllll}
\hline FII & 4 & 3 & 5 & 2 \\
FXI1 & 4 & 3 & 5 & 2 \\
FXII1 & 4 & 3 & 5 & 2 \\
FVI1 & 4 & 2 & 2 & 2 \\
FVIII1 & 4 & 2 & 2 & 2 \\
FIX1 & 4 & 2 & 2 & 2 \\
FX1 & 4 & 3 & 5 & 2 \\
FXIII1 & 4 & 3 & 5 & 2 \\
FIV1 & 4 & 3 & 5 & 2 \\
FVII1 & 4 & 2 & 5 & 2 \\
FI2 & 4 & 3 & 5 & 2 \\
FI1+3 & 4 & 3 & 5 & 2 \\
FIII & 4 & 3 & 5 & 2 \\
FV & 4 & 2 & 2 & 2
\end{tabular}

CAG GGC

$\begin{array}{lll}45 & 18 & 2 \\ 41 & 18 & 2 \\ 42 & 18 & 2 \\ 42 & 18 & 2 \\ 45 & 18 & 2 \\ 47 & 18 & 2 \\ 47 & 18 & 2 \\ 45 & 18 & 2 \\ 44 & 18 & 2 \\ 44 & 18 & 2 \\ 43 & 18 & 2 \\ 44 & 18 & 2 \\ 45 / 46 & 18 & 2 \\ 44 / 45 & 18 & 2\end{array}$

$\begin{array}{rrrrrr}4 & 6 & 7+ & 11 & 8 & 10 \\ 4 & 6 & 7+ & 11 & 8 & 10 \\ 4 & 6 & 7+ & 11 & 8 & 10 \\ 4 & 6 & 7+ & 11 & 8 & 10 \\ 4 & 6 & 7+ & 11 & 8 & 10 \\ 4 & 6 & 7+ & 11 & 8 & 10 \\ 4 & 6 & 7+ & 11 & 7 & \\ 4 & 6 & 7+ & 11 & 7 & 7 \\ 4 & 6 & 7+ & 11 & 7 & 7 \\ 4 & 6 & 7+ & 11 & 7 & \\ 4 & 6 & 7+ & 11 & 7 & \\ 4 & 6 & 7+ & 2 & 3 & \\ 4 & 6 & 7+ & 5 & 8 & 9 \\ 4 & 5 & 3+ & 11 & 7 & 9\end{array}$

$\begin{array}{rrllr}10 & 1 & 2 & 3 & 3 \\ 10 & 1 & 2 & 3 & 3 \\ 10 & 1 & 2 & 3 & 3 \\ 10 & 1 & 2 & 3 & 3 \\ 10 & 1 & 2 & 3 & 3 \\ 10 & 1 & 2 & 3 & 3 \\ 7 & 4 & 7 & 2 & 13 \\ 7 & 4 & 7 & 2 & 13 \\ 7 & 4 & 7 & 2 & 13 \\ 7 & 4 & 7 & 2 & 13 \\ 7 & 4 & 7 & 2 & 13 \\ 7 & 4 & 1 & 2 & 1 \\ 9 & 1 & 1 & 1 & 7 \\ 9 & 2 & 4 & 2 & 3\end{array}$

$\begin{array}{lrrrrrrrrrrrrrrrrrrr}\text { SII1 } & 2 & 2 & 3 & 2 & 3 & 42 & 18 & 2 & 4 & 10 & 6- & 11 & 7 & 8 & 2 & 6 & 1 & 13 \\ \text { SIII1 } & 2 & 2 & 3 & 2 & 3 & 44 & 18 & 2 & 4 & 10 & 6- & 11 & 7 & 8 & 2 & 6 & 1 & 13 \\ \text { SVI1 } & 2 & 2 & 3 & 2 & 3 & 43 & 18 & 2 & 4 & 10 & 6- & 11 & 7 & 8 & 2 & 6 & 1 & 13 \\ \text { SVII1 } & 4 & 2 & 3 & 2 & 3 & 47 & 18 & 2 & 4 & 10 & 6- & 11 & 7 & 8 & 2 & 6 & 1 & 13 \\ \text { SX1 } & 2 & 2 & 3 & 2 & 3 & 46 & 18 & 2 & 4 & 10 & 6- & 11 & 7 & 8 & 2 & 6 & 1 & 13 \\ \text { SVIII1 } & 4 & 2 & 2 & 2 & 3 & 44 & 18 & 2 & 4 & 10 & 6- & 11 & 8 & 7 & 2 & 4 & 1 & 2 \\ \text { SIX2 } & 2 & 2 & 4 & 2 & 3 & 45 & 18 & 2 & 4 & 10 & 6- & 11 & 7 & 8 & 2 & 6 & 1 & 13 \\ \text { SIX1 } & 2 & 2 & 3 & 2 & 3 & 45 & 18 & 2 & 4 & 10 & 6- & 2 & 3 & 7 & 3 & 5 & 1 & 13 \\ \text { SV1 } & 2 & 2 & 3 & 2 & 3 & 41 & 18 & 2 & 4 & 3 & 4- & 11 & 7 & 10 & 3 & 4 & 3 & 3 \\ \text { SI1 } & 4 & 2 & 4 & 2 & 3 & 38 & 18 & 2 & 4 & 6 & 7+ & 11 & 8 & 10 & 1 & 2 & 3 & 3 \\ \text { SIV } & 4 & 2 & 2 & 2 & 3 & 45 & 18 & 1 & 4 & 5 & 4- & 5 & 3 & 9 & 3 & 1 & 2 & 8\end{array}$

$\begin{array}{lrlllllllllllllllll}\text { DIV1 } & 4 & 7 & 5 & 3 & 3 & 44 & 18 & 2 & 6 & 5 & 7+ & 5 & 3 & 7 & 3 & 5 & 3 & 4 \\ \text { DVI1 } & 4 & 7 & 5 & 3 & 3 & 44 & 18 & 2 & 6 & 5 & 7+ & 5 & 3 & 7 & 3 & 5 & 3 & 4 \\ \text { DVII1 } & 4 & 7 & 5 & 3 & 3 & 49 & 18 & 2 & 6 & 5 & 7+ & 5 & 3 & 7 & 3 & 5 & 3 & 4 \\ \text { DVIII1 } & 4 & 7 & 5 & 3 & 3 & 50 & 18 & 2 & 6 & 5 & 7+ & 5 & 3 & 7 & 3 & 5 & 3 & 4 \\ \text { DXI1 } & 4 & 7 & 5 & 3 & 3 & 49 & 18 & 2 & 6 & 5 & 7+ & 5 & 3 & 7 & 3 & 5 & 3 & 4 \\ \text { DI1 } & 4 & 3 & 7 & 3 & 3 & 48 & 18 & 2 & 6 & 5 & 7+ & 5 & 3 & 7 & 3 & 5 & 2 & 4 \\ \text { DX1 } & 4 & 3 & 7 & 3 & 3 & 49 & 18 & 2 & 6 & 5 & 7+ & 5 & 3 & 7 & 3 & 5 & 2 & 4 \\ \text { DIX1 } & 4 & 2 & 4 & 6 & 7 & 47 & 18 & 2 & 6 & 5 & 5+ & 5 & 1 & 8 & 1 & 1 & 2 & 12 \\ \text { DII1 } & 4 & 7 & 5 & 3 & 3 & 51 & 18 & 2 & 6 & 6 & 9- & 5 & 3 & 7 & 3 & 5 & 2 & 13 \\ \text { DIII1 } & 4 & 7 & 5 & 3 & 3 & 41 & 18 & 2 & 6 & 4 & 7+ & 5 & 3 & 7 & 3 & 5 & 3 & 4 \\ \text { DV1 } & 4 & 7 & 5 & 3 & 3 & 47 & 18 & 2 & 6 & 4 & 6- & 5 & 7 & 8 & 2 & 7 & 2 & 12 \\ \text { DXII1 } & 4 & 3 & 3 & 3 & 3 & 45 & 18 & 2 & 4 & 8 & 5 & 5 & 2 & 4 & 3 & 5 & 2 & 13 \\ \text { NI } & 4 & 7 & 6 & 2 & 3 & 44 & 18 & 2 & 4 & 6 & 7+ & 11 & 8 & 10 & 1 & 2 & 3 & 3 \\ \text { NII } & 4 & 2 & 2 & 2 & 3 & 43 / 44 / 18 & 5 & 4 & 5 & 2- & 11 & 3 & 7 & 2 & 7 & 2 & 4\end{array}$

$\begin{array}{llllll}\text { Marker } & 8062 & 8032 & \text { ALAS2 } & 1213 & 1194 \\ \text { Allele } & & & & & \\ 1 & 98 & 198 & 167 & 244 & 267 \\ 2 & 94 & 192 & 165 & 240 & 283 \\ 3 & 90 & 200 & 163 & 236 & 269 \\ 4 & 92 & 190 & 161 & 242 & 275 \\ 5 & 96 & 196 & 159 & 238 & 263 \\ 6 & 88 & 188 & 157 & 234 & 261 \\ 7 & & 194 & 155 & 232 & 277 \\ 8 & & 202 & 153 & 230 & 265 \\ 9 & & 204 & 149 & & 271 \\ 10 & & & 169 & & \end{array}$

\begin{tabular}{|c|c|c|c|c|c|c|c|c|c|}
\hline $1111 \quad 135$ & 1125 & 981 & 1689 & 339 & 106 & 1216 & 1275 & 453 & 1690 \\
\hline 238 & 138 & 199 & 161 & 214 & 107 & 246 & 212 & 170 & 144 \\
\hline 236 & 136 & 198 & 159 & 212 & 105 & 244 & 210 & 168 & 142 \\
\hline 234 & 134 & 195 & 157 & 210 & 103 & 248 & 216 & 166 & 140 \\
\hline 232 & 132 & 194 & 155 & 208 & 101 & 250 & 214 & 164 & 138 \\
\hline 230 & 130 & 191 & 153 & 206 & 99 & & 206 & 160 & 136 \\
\hline 228 & 128 & 190 & 151 & 204 & 97 & & 220 & 172 & 134 \\
\hline 226 & 126 & 187 & 149 & 202 & 95 & & 218 & 150 & 132 \\
\hline & 124 & 186 & 147 & 200 & 93 & & 208 & & 130 \\
\hline & 122 & 183 & 145 & & 91 & & 222 & & 128 \\
\hline & 144 & 182 & 143 & & 89 & & & & 126 \\
\hline & & 202 & 167 & & 87 & & & & 124 \\
\hline & & & 169 & & & & & & 16 \\
\hline & & & 171 & & & & & & 148 \\
\hline & & & 165 & & & & & & 50 \\
\hline & & & & & & & & & \\
\hline
\end{tabular}


12 Danish families shared the same DXS1213-DXS135 haplotype. However they harboured al lele 3 for the locus DXS1213 instead of 2, and allele 6 for the locus DXS135 instead of 4. In the Finnish, Swedish and Danish SBMA families a countryspecific common DXS8062-DXS1690 founder haplotype was detected (Table 1, shaded areas). Moreover, all the Finnish, Swedish, Danish and Norwegian families shared the same 18GGC repeats allele, and $92 \%$ of the families had the DXS1194-DXS1111 haplotype 3-18-2 (Table1). One Swedish patient, SI1, and one Norwegian family, NI, had the DXS1213-DXS1690 haplotype 2-3-18-2-4-6-7 + $-11-8-10-1-2-3-3$ (15.6cM), which is common among Finnish SBMA families (Table1). All the controls showed different haplotypes (data not shown).

The 142 control chromosomes displayed a CAG repeat number ranging from 15 to 29 (Figure 2). The number of CAG repeats in the patients ranged from 38 to 51 (Figure3). The range of CAG repeat numbers in Finnish patients was $41-47$, with the mean of $44.3 \pm 1.7$. In Swedish patients the range of repeat numbers was $38-47$, mean $43.6 \pm 2.5$. The Danish patients displayed $41-51$ CAG repeats, mean $47 \pm 3$ (Figure3). The two Norwegian families had 43-45 repeats (Table1). The difference between the CAG ranges of the Finnish and the Swedish patients was not statistically significant, but the Danish patients differed significantly from the others $(P=0.003)$.

The intragenic GGC repeat sizes ranged from 11 to 21 in controls, with 17 being the most common allele, present in $48 \%$ of the Finnish, $55 \%$ of the Swedish and $70 \%$ of the Danish controls (Figure4). All the Finnish, Swedish, Danish and Norwegian patients had $18 \mathrm{GGC}$ repeats, also present in $26 \%$ of the Finnish, $27 \%$ of the Swedish and $15 \%$ of the Danish controls $(P=0.0001)$, suggesting linkage disequilibrium between the CAG repeat expansion and the GGC microsatel lite.

Linkage disequilibrium was also seen between the mutation and the locus DXS1111, since allele 2 was present in $94.5 \%$ of the SBM A families and in only $40 \%$ of the Finnish, $18 \%$ of the Swedish and $25 \%$ of the Danish controls $(P=0.0001)$. All the Finnish, Swedish and Norwegian families shared the same allele 4 for the locus DXS135, which was present in $41 \%(P=0.004)$ and $50 \%(P=0.002)$ of the respective controls. However, allele4 was seen in only one Danish family, the remaining 11 (92\%) carrying allele6 present in $20 \%$ of the Danish controls $(P=0.0003)$. All the Finnish, Swedish and Norwegian families shared allele2 in DXS1213, present in 62\% $(P=0.05)$ and 50\% ( $P=0.03)$ of the respective controls, and 11 (92\%) of the Danish families had allele 3 , present in $10 \%$ of the Danish controls $(P=0.08)$. Allele 3 of the locus DXS1194 was present in all except one (97\%) patients, and also in $66 \%$ of the controls ( $P=0.0002)$.

The maximum likelihood peak in the age estimation using the DMLE program (see Materials and methods for assumptions) for the closest recombinant flanking loci, ALAS2 and
CAG Finnish controls

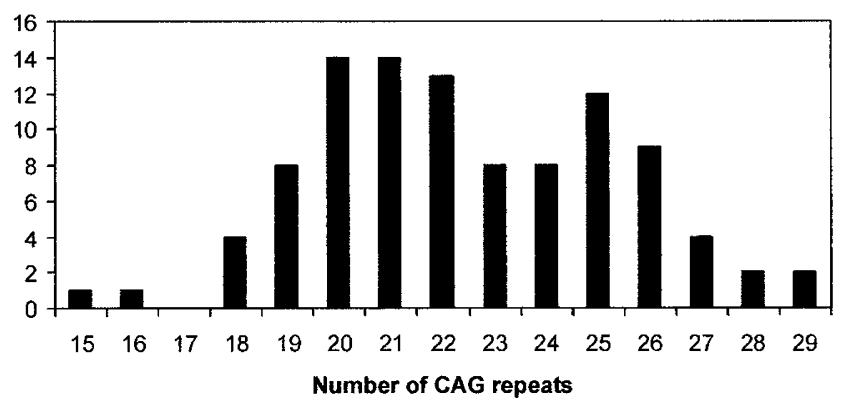

CAG Swedish controls

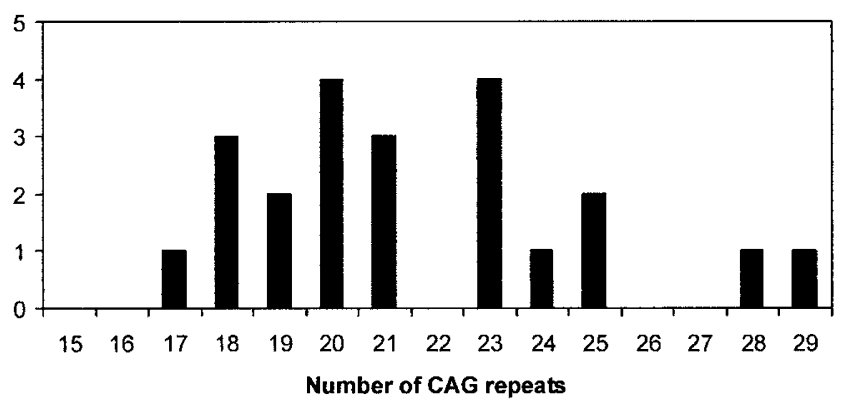

CAG Danish controls

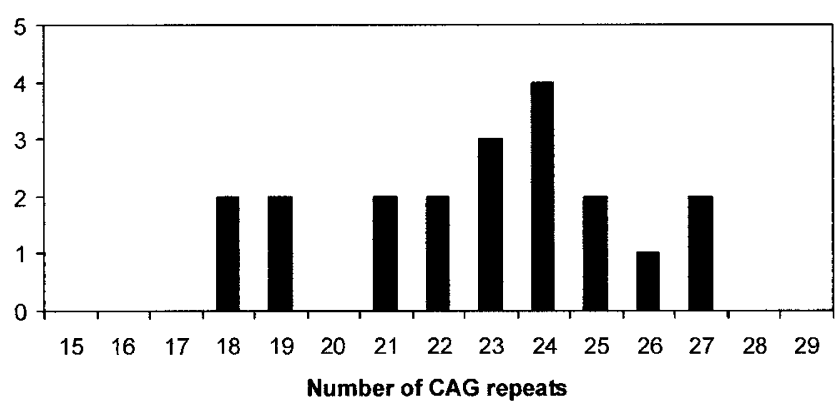

Figure 2 Numbers of CAG repeats in the controls.

DXS1125, in the Finnish SBMA families was obtained at 20 generations for each (data not shown).

\section{Discussion}

All the Finnish and 9 of the 10 Swedish SBMA chromosomes harboured an identical haplotype spanning a $4.8 \mathrm{cM}$ region (GDB) (Table1) on chromosome Xq11-q12. This suggests a common founder haplotype for these patients. The different alleles of the loci DXS1213 and DXS135 in the Danish SBMA patients compared to those carried by Finnish and Swedish patients indicate a more ancient separation. The DXS1194DXS1111 haplotype shared by $92 \%$ of the families (Table 1 ) composes a potential Scandinavian founder haplotype. There was also evidence of migration between countries, since SI, 


\section{CAG Finnish patients}

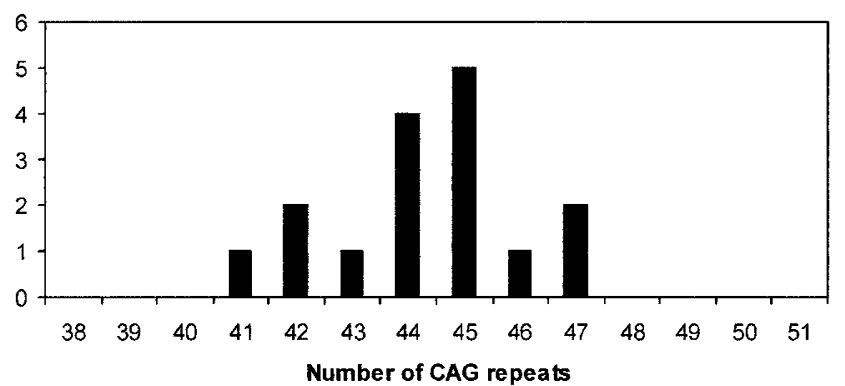

CAG Swedish patients

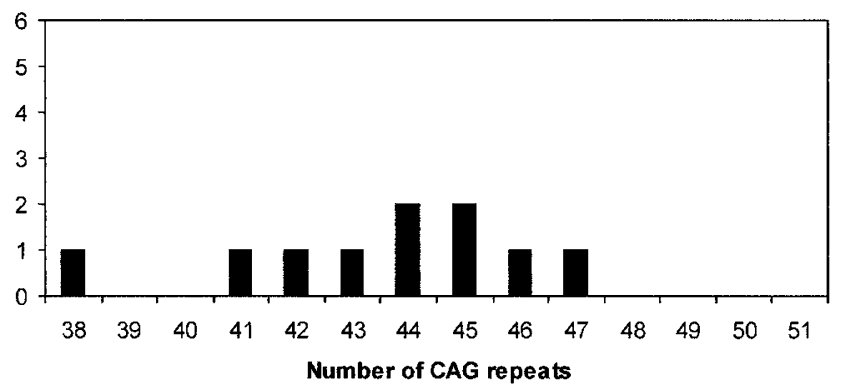

CAG Danish patients

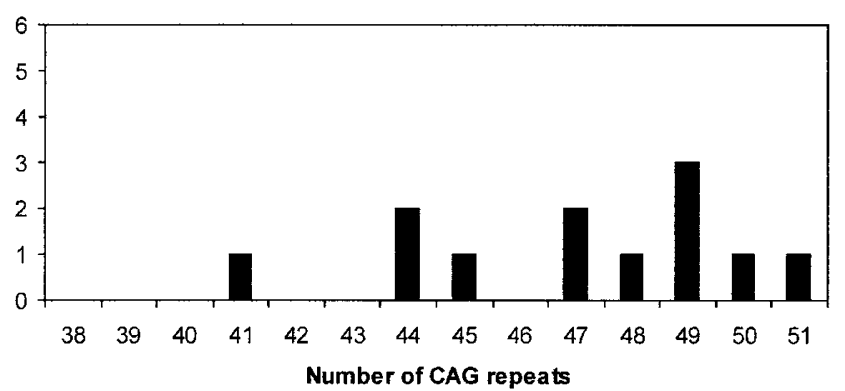

Figure 3 Numbers of CAG repeats in the patients.

$\mathrm{NI}$ and six Finnish SBM A families shared the same haplotype for DXS1213-DXS1690.

The ranges of the CAG repeat sizes of the Finnish, Swedish and Norwegian patients (Figure3, Table1) were quite similar, 44 and 45 repeats being the most common. The Danish patients had a somewhat wider range with slightly longer repeats, 49 repeats being the most common. If the Danish mutation had a more ancient origin, it would have had more time to develop a wider range. The comparable ranges shown here in the Finnish, Swedish and Norwegian patients suggest a more recent separation between these.

In Japanese SBMA patients linkage disequilibrium was found between the CAG repeat expansion and the GGC repeat, indicating a founder effect. ${ }^{13}$ Nevertheless, in the Japanese patients the linkage disequilibrium was not as complete as in our study, where all Nordic patients shared the same GGC repeat number. A founder effect was also suggested to be responsible for the clustering of SCA6 families in the Nordrhein-Westfalia area in Germany, where the majority of the families shared allelic characteristics of three markers around the associated gene. ${ }^{15} \mathrm{~A}$ founder effect has also been reported in SCA $1,{ }^{16}$ SCA $2^{17,18}$ and SCA3 (MachadoJoseph disease). ${ }^{19}$

Twelve of the 13Finnish SBMA families originated from the Vaasa region on the western coast of Finland. One patient (FVIIII) lived in Southern Finland. The fairly restricted area from which the SBMA patients originated and the fact that 11 of these 13 families were Swedish-speaking supports the theory of a common ancestor. Nevertheless, genealogical

GGC Finnish controls

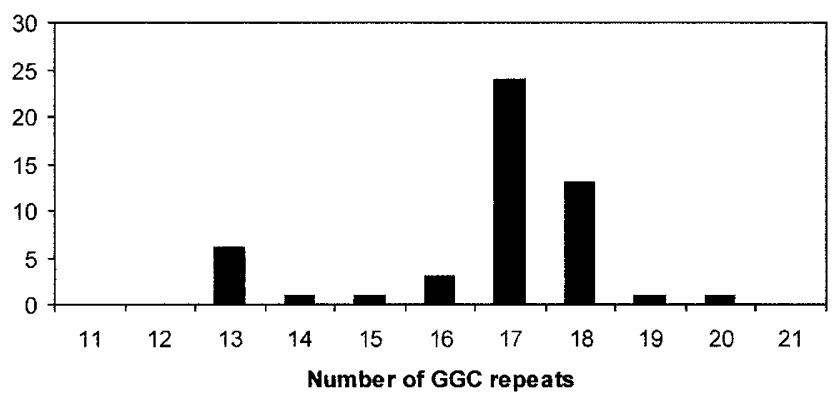

GGC Swedish controls

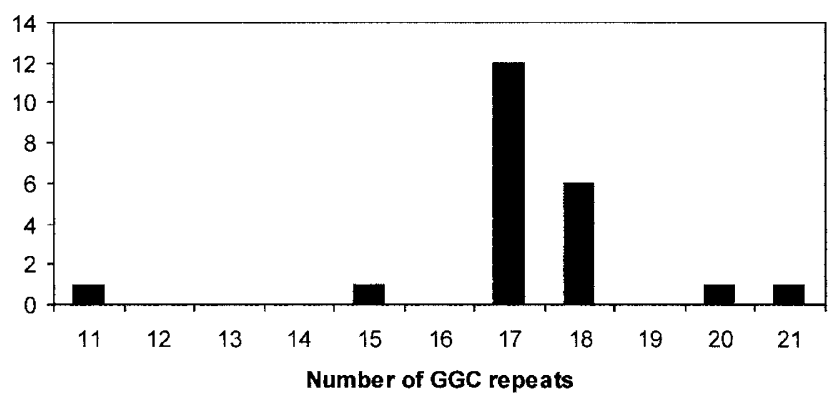

GGC Danish controls

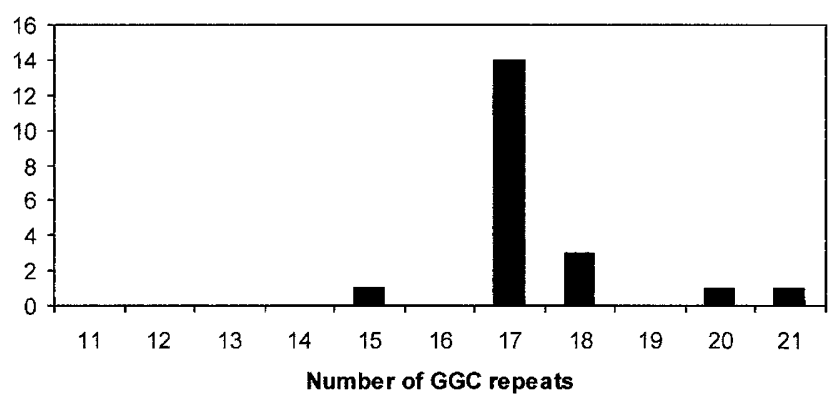

Figure 4 Numbers of GGC repeats in the controls. 
studies have not revealed any definite genetic links between these families over the past 200 years.

The Vaasa region in western Finland was inhabited during the Stone, Bronze and Iron Ages. Some later resettlement from Sweden is documented from mediaeval times, along with the expansion of Christianity, and later until the 18th century. Since many of the Swedish patients come from the areas located across the Gulf of Bothnia from the Vaasa region, and because of the common language, a common genetic background for the Finnish and the Swedish SBMA patients is indicated.

The maximum likelihood peak for ALAS2 and DXS1125 of the Finnish SBMA patients obtained by DMLE, 20 generations, would indicate the introduction of the SBMA mutation into the Finnish Swedish-speaking coastal population around 1400-1500. However, whilst the estimate seems close to the truth, it has very wide confidence limits and, on the other hand, is based for simplicity on assumptions that almost certainly are not the whole truth.

The fact that all the Scandinavian patients with SBMA carry the same intragenic GGC allele18 in addition to other similarities in allele distribution suggests an ancient Scandinavian founder effect. There also seems to be a specific more extended sub-founder haplotype in each country. Later on, we plan to elucidate the possible similarities between patients from around the world, and to find out whether multiple founder effects exist.

\section{Acknowledgements}

We thank the patients for their co-operation. We are grateful to $\mathrm{Dr}$ Kristiina Aittomäki for providing the DNA from the patient living in Southern Finland. The technical assistance of $\mathrm{Mrs}$ Ilona Carlsson-Suvanto, Ms Emma Metsämäki and Mrs Hanna Kukkula is acknowledged. The statistical assistance of Mr Tommi Viitanen and the clerical assistance of Mrs Pia Tahvanainen is also greatly appreciated. Special thanks are due to Dr Jürgen Häussler for kindly providing the sequenced reference sample for the GGC repeats. This work was supported by the University of Turku Foundation, the Tampere University Hospital Research Foundation, the Finnish Neuromuscular Disease Research Foundation and the Sigrid Jusélius Foundation.

\section{References}

1 Kennedy WR, Alter M, Sung J: Progressive proximal spinal and bulbar muscular atrophy of late onset: A sex-linked recessive trait. Neurology 1968; 18: 671-680.
2 Udd B, Juvonen V, Hakamies L et al: High prevalence of Kennedy's disease in Western Finland - is the syndrome underdiagnosed? Acta Neurol Scand 1998; 98: 128-133.

3 La Spada AR, Wilson EM, Lubahn DB, Harding AE, Fischbeck KH: Androgen receptor gene mutations in $\mathrm{X}$-linked spinal and bulbar muscular atrophy. Nature 1991; 352: 77-79.

4 Merry DE, Kobayashi Y, Bailey CK, Taye AA, Fischbeck KH: Cleavage, aggregation and toxicity of the expanded androgen receptor in spinal and bulbar muscular atrophy. Hum Mol Genet 1998; 7: 693-701.

5 Burright EN, Orr HT, Clark HB: Mouse models of human CAG repeat disorders. Brain Pathol 1997; 7: 965-977.

6 Butler R, Leigh PN, McPhaul MJ, Gallo J-M: Truncated forms of the androgen receptor are associated with polyglutamine expansion in X-linked spinal and bulbar muscular atrophy. Hum Mol Genet 1998; 7: 121-127.

7 Pearson CE, Sinden RR: Trinucleotide repeat DNA structures: dynamic mutations from dynamic DNA. Curr Opin Struct Biol 1998; 8: 321-330.

8 Koob MD, Moseley ML, Schut LJ et al: An untranslated CTG expansion causes a novel form of spinocerebellar ataxia (SCA8). Nat Genet 1999; 21: 379-384.

9 Holmes SE, O'Hearn EE, Mclnnis MG et al: Expansion of a novel CAG trinucleotide repeat in the 5 ' region of PPP2R2B is associated with SCA12. Nat Genet 1999; 23: 391-392.

10 Igarashi S, Koide R, Shimohata $T$ et al: Suppression of aggregate formation and apoptosis by transglutaminase inhibitors in cells expressing truncated DRPLA protein with an expanded polyglutamine stretch. Nat Genet 1998; 18: 111-117.

11 Abdullah AAR, Trifiro MA, Panet-Raymond V et al: Spinobulbar muscular atrophy: polyglutamine-expanded androgen receptor is proteolytically resistant in vitro and processed abnormally in transfected cells. Hum M ol Genet 1998; 7: 379-384.

12 Sleddens HFBM, Oostra BA, Brinkmann AO, Trapman J: Trinucleotide (GGN) repeat polymorphism in the human androgen receptor (AR) gene. Hum Mol Genet 1993; 2: 493.

13 Tanaka F, Doyu M, Ito $Y$ et al: Founder effect in spinal and bulbar muscular atrophy (SBMA). Hum Mol Genet 1996; 5: 1253-1257.

14 Rannala B, Slatkin M: Likelihood analysis of disequilibrium mapping, and related problems. Am J Hum Genet 1998; 62: 459-473.

15 Dichgans M, Schöls L, Herzog J et al: Spinocerebellar ataxia type6: Evidence for a strong founder effect among German families. Neurology 1999; 52: 849-851.

16 Wakisaka A, Sasaki H, Takada A et al: Spinocerebellar ataxia 1 (SCA1) in the Japanese in Hokkaido may derive from a single common ancestry. J Med Genet 1995; 32: 590-592.

17 Hernandez A, Magarino C, Gispert Set al: Genetic mapping of the spinocerebellar ataxia2 (SCA2) locus on chromosome12q23q24.1. Genomics 1995; 25: 433-435.

18 Mizushima K, Watanabe M, Kondo I et al: Analysis of spinocerebellar ataxia type2 gene and haplotype analysis: (CCG)1-2 polymorphism and contribution to founder effect. J Med Genet 1999; 36: 112-114.

19 Endo K, Sasaki H, Wakisaka A et al: Strong linkage disequilibrium and haplotype analysis in Japanese pedigrees with MachadoJoseph disease. Am J Med Genet 1996; 67: 437-444. 